\title{
Les résultats des campagnes de mesures de radon et facteurs explicatifs *
}

\author{
A. RANNOU ${ }^{\star \star}$, G. TYMEN***
}

\begin{abstract}
RÉSUMÉ
Des mesures de radon ont été effectuées à l'aide de détecteurs passifs dans plus de 2500 maisons, réparties dans 36 départements français. Les 3006 résultats obtenus indiquent une moyenne arithmétique égale à $115 \mathrm{~Bq} \cdot \mathrm{m}^{-3}$, et une moyenne géométrique égale à $62 \mathrm{~Bq} \cdot \mathrm{m}^{-3}$, avec un écart-type de 2,74. En corrigeant la distribution des données pour tenir compte de la densité de population ou de logements à l'échelle du département, les moyennes arithmétique et géométrique sont évaluées à $62 \mathrm{~Bq} \cdot \mathrm{m}^{-3}$ et $41 \mathrm{~Bq} \cdot \mathrm{m}^{-3}$ respectivement. L'analyse des données montre une influence de la nature du sous-sol prépondérante par rapport à d'autres facteurs explicatifs: niveau de la pièce dans la maison, type de pièce, matériaux de construction, ou âge de la maison.
\end{abstract}

\section{ABSTRACT}

Radon was measured with passive detectors in over 2500 houses distributed throughout 36 french "departements". The 3006 results showed an arithmetic mean of $115 \mathrm{~Bq} \cdot \mathrm{m}^{-3}$, and a geometric mean of $62 \mathrm{~Bq} \cdot \mathrm{m}^{-3}$, with a standard deviation of 2.74. By correcting data distribution to account for population or dwelling densities at the department level, arithmetic and geometric means were evaluated at 62 and $41 \mathrm{~Bq} \cdot \mathrm{m}^{-3}$ respectively. An analysis of the data showed the prevailing effect of the subsoil as compared to other factors: room level in the dwelling, building materials, age of the dwelling.

- Communication présentée lors de la réunion SFRP "Exposition au radon dans les habitations: aspects techniques et sanitaires", Paris, 10-11 janvier 1989.

** Commissariat à l'énergie atomique, Institut de protection et de sûreté nucléaire, DPS/SEAPS, BP 6, 92265 Fontenay-aux-Roses Cedex.

*** Faculté des sciences de Brest, Laboratoire de physique des aérosols et de radioactivité atmosphérique, 6, avenue Victor Le Gorgeu, 29287 Brest Cedex. 


\section{INTRODUCTION}

Bien qu'il y ait eu une prise de conscience assez générale dans beaucoup de pays, notamment en Europe, pour vouloir mieux connaître l'exposition du public au radon dans les habitations, relativement peu de mesures ont été faites avant les années 1980. Ceci ne signifie évidemment pas que les expositions moyennes estimées jusqu'alors pour l'ensemble des populations aient été mauvaises ou même fausses, mais simplement que la variabilité géographique était souvent peu ou mal connue et la distribution statistique des expositions individuelles insuffisamment précise.

II fut assez rapidement reconnu qu'il pouvait y avoir une grande différence de niveaux d'un pays à l'autre et que le problème ne se posait pas partout de la même façon. Ceci justifiait que, dans chaque pays, un programme de mesure soit développé pour prendre en compte l'extrême diversité des situations liées aux facteurs climatiuues, géologiques, architecturaux ou encore aux habitudes de vie spécifiques à chaque pays. Alors que les objectifs recherchés étaient les mêmes, les méthodologies adoptées dans les différents pays allaient, au contraire, se différencier par:

- la méthode d'échantillonnage,

- les procédures expérimentales: technique(s) mise(s) en œuvre et protocole(s).

II est important que la méthodologie employée soit connue si l'on veut correctement interpréter les résultats obtenus et les comparer à d'autres bilans nationaux.

\section{MÉTHOdOLOGIE D'ÉCHANTILLONNAGE}

Toutes les méthodes d'échantillonnage utilisées - que celui-ci soit réalisé à une échelle nationale ou régionale - reposent sur le principe du volontariat. Différentes approches ont été retenues:

- tirage aléatoire d'individus d'une population donnée en respectant la représentativité de l'échantillon par rapport à la distribution réelle de la population (sondage d'un échantillon dit représentatif) - c'est la méthode choisie par exemple en Irlande [5], en Angleterre [13], en République fédérale d'Allemagne [7] ou encore en Suède [11] (dans ce cas, l'échantillon bien choisi peut être limité en nombre);

- tirage d'individus à partir d'une liste de volontaires, obtenue à la suite d'une campagne de presse, comme aux Pays-Bas [8];

- échantillonnage au hasard, selon les opportunités, -c'est le cas des études menées aux Etats-Unis de façon relativement indépendante par chacun des États.

La campagne de mesure du radon dans les habitations, menée en France par le département de Protection sanitaire du Commissariat à l'énergie atomique (CEA), a débuté en 1983. Cette campagne est venue se greffer 
sur le programme déjà plus ancien conduit pour établir une cartographie des niveaux d'exposition gamma d'origine tellurique en France [6]. Dans chaque département étudié, une liste des communes avec le nombre de points de mesure souhaités pour chacune d'entre elles est établie, en tenant compte de la densité de population. Le cas échéant, des points supplémentaires sont recherchés pour éviter de trop grosses lacunes dans le maillage géographique. Cette liste est transmise aux autorités préfectorales qui ont en charge de trouver les volontaires avec le support de la Direction départementale de la Protection civile. Les volontaires sont recherchés le plus souvent parmi le personnel des administrations: sapeurs-pompiers, gendarmes, policiers, maires et employés municipaux, etc., mais aussi dans certains corps de métier, dont l'enseignement par exemple.

La méthode d'échantillonnage ainsi en cours pour les mesures d'exposition gamma a donc été adoptée pour les mesures de radon, leur nombre étant, cependant, plus limité au cours des premières années (50 puis 100 mesures par département). Compte tenu de l'influence du mode de vie et du type de construction sur le niveau de radon, il est demandé que les mesures soient évitées dans les locaux professionnels et ne concernent que des pièces principales d'habitation.

Dans les études régionales plus spécifiques, les volontaires ont été recherchés dans la seule profession des pharmaciens (Dordogne et Limousin) ou par l'intermédiaire des mairies (départements bretons). Cette approche plus directe, mais aussi plus lourde, permet un échantillonnage en principe mieux décentralisé.

\section{PROCÉduRES EXPÉRIMENTALES}

Nous ne rappellerons ici que très brièvement les techniques mises en œuvre, déjà largement exposées par ailleurs [6].

Le dosimètre passif de type ouvert utilisé repose sur le principe de la détection solide de traces par un film (LR 115, Kodak-Pathé) sensible aux particules alpha émises par les atomes de radon et de ses descendants à vie courte. Sa sensibilité égale à 1,3 traces $/ \mathrm{kBq} . \mathrm{h} \cdot \mathrm{m}^{-3}$ lui confère une limite de détection voisine de 6 Bq. $\mathrm{m}^{-3}$ pour une exposition de $30 \mathrm{j}$. L'intercomparaison du dosimètre par rapport à des méthodes de référence montre une erreur maximale de $\pm 20 \%$ dans la gamme de niveaux de radon généralement rencontrés dans les habitations [9].

Exposé pendant 1 mois au cours des premières années, le dosimètre passif est aujourd'hui exposé pendant 2 mois. La mise en place des dosimètres se fait généralement soit au début du printemps, soit au début de l'automne. Ces deux époques de l'année ont l'avantage d'intégrer des concentrations moyennes comprises entre celles maximales observées en hiver et celles minimales observées en été.

Le dosimètre actif utilisé a été mis au point au CEA [1]. C'est un appareil dérivé de celui que portent les mineurs de mines d'uranium en France. 
Une pompe effectue un prélèvement des descendants radioactifs du radon ${ }^{(222} R n$ et $\left.{ }^{220} R n\right)$, contenus dans l'air. Une fois collectés sur le filtre, ceux-ci se désintègrent au travers d'un système de collimateurs et d'écrans d'épaisseur choisie pour sélectionner les particules alpha émises en fonction de leur énergie. L'appareil permet ainsi le calcul de la concentration d'énergie potentielle alpha (EPA) dans l'air pour chacune des deux familles du radon.

La durée nominale de fonctionnement est de 3 semaines environ.

\section{RÉSULTATS}

La concentration en radon a été mesurée à ce jour dans plus de 2500 maisons réparties dans 35 départements et en lle-de-France. Du fait des mesures doubles voulues dans le cadre des études régionales, c'est un total de 3000 mesures qui peuvent être analysées aujourd'hui.

\subsection{Aspects généraux des résultats}

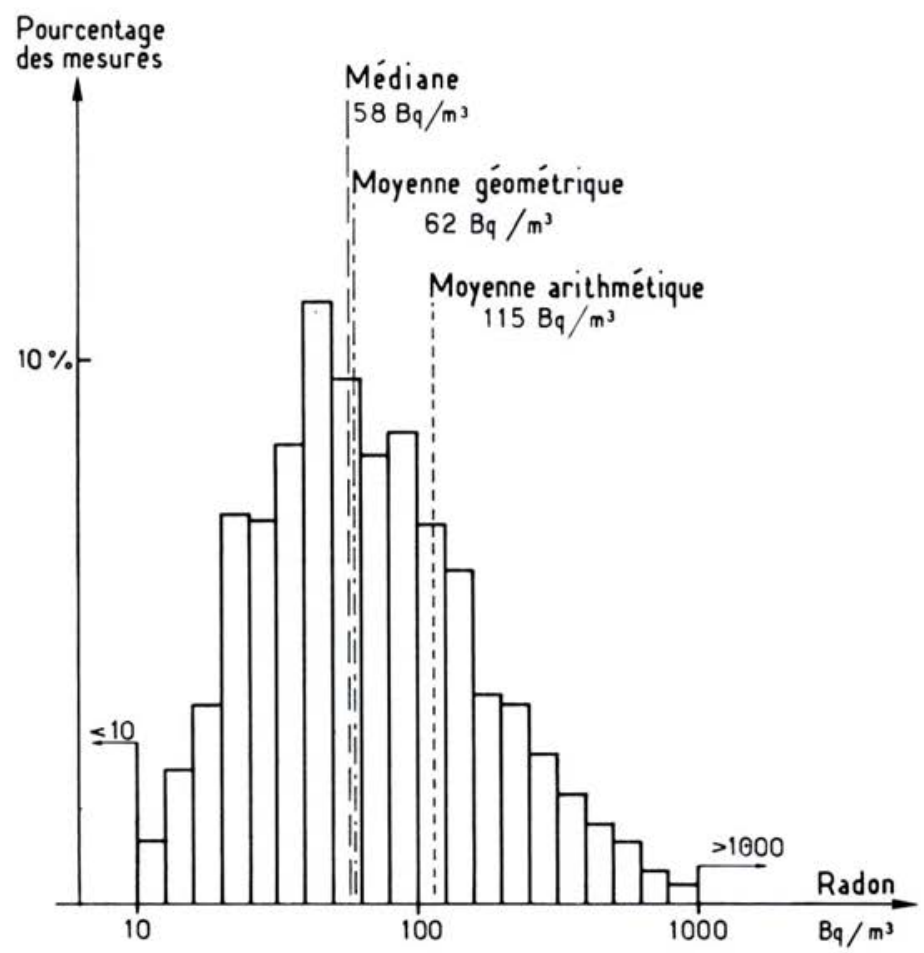

Fig. 1. - Histogramme de répartition des mesures de concentration en radon dans les habitations, d'après 3006 résultats. 
L'histogramme de la répartition des résultats de mesure (fig. 1) montre la grande étendue des niveaux rencontrés. Les valeurs s'échelonnent sur quatre ordres de grandeur entre les minimums proches de la limite de détection et un maximum trouvé égal à $4687 \mathrm{~Bq} \cdot \mathrm{m}^{-3}$. La grande étendue observée explique les écarts importants entre les différentes moyennes calculées. La moyenne arithmétique $\left(115 \mathrm{~Bq} \cdot \mathrm{m}^{-3}\right)$ est très surestimée par rapport à la moyenne géométrique $\left(62 \mathrm{~Bq}^{-3} \mathrm{~m}^{-3}\right)$ et la valeur médiane (58 Bq. $\mathrm{m}^{-3}$ ) en raison des fortes valeurs. La distribution s'apparente assez bien à celle d'une loi de type log-normale. Bien entendu, cet échantillon n'a pas de valeur statistique et ne donne pas une image réaliste de la situation, que ce soit pour les logements ou pour la population.

Le nombre de mesures, plus important dans les régions granitiques, déplace la distribution des résultats vers les valeurs élevées et augmente ainsi artificiellement le niveau moyen. L'image donnée peut cependant être corrigée en tenant compte du nombre de mesures effectuées dans chaque département et de son nombre de logements ou de sa population. Pour cela, il suffit de pondérer, pour chaque département, le pourcentage de résultats figurant dans chacun des intervalles de mesure choisis par le nombre d'habitants ou de logements du département.

La correction en population ainsi faite conduit à l'histogramme de la figure 2. Une répartition très voisine est obtenue dans le cas où la correction est faite en logements. Les moyennes géométrique et arithmétique calculées sont égales à $41 \mathrm{~Bq} \cdot \mathrm{m}^{-3}$ et $62 \mathrm{~Bq} \cdot \mathrm{m}^{-3}$ respectivement, quelle que soit la correction choisie; la moyenne de la distribution (médiane) est égale à $33 \mathrm{~Bq} \cdot \mathrm{m}^{-3}$. Selon cette approche, près de $5 \%$ de la population serait exposée à une concentration en radon supérieure à 200 Bq. $\mathrm{m}^{-3}$ (soit 100 Bq. $\mathrm{m}^{-3}$ de descendants du radon à l'équilibre) et $1,5 \%$ environ de la population à plus de $400 \mathrm{~Bq} \cdot \mathrm{m}^{-3}$ (soit $200 \mathrm{~Bq} \cdot \mathrm{m}^{-3}$ de descendants du radon à l'équilibre). Selon les mêmes hypothèses, $3,5 \%$ des logements des régions étudiées indiqueraient un niveau de concentration excédant 200 Bq. $\mathrm{m}^{-3}$ et $1,5 \%$ celui de 400 Bq. $\mathrm{m}^{-3}$.

Même si ces estimations restent grossières et reposent sur l'étude de seulement un département français sur trois, il est probable que ces ordres de grandeur soient à peu près corrects pour la France entière.

\subsection{Variations géographiques}

L'analyse des résultats par département met en évidence des variations géographiques significatives. Cette constatation s'explique sans peine lorsqu'on regarde les caractéristiques géologiques du sol. Les départements où les niveaux moyens de concentration sont les plus bas $\left(\leqslant 30 \mathrm{~Bq} \cdot \mathrm{m}^{-3}\right)$ sont ceux dont les terrains sont à dominante sédimentaire: Bouches-du-Rhône (moyenne géométrique $\mathrm{m}_{\mathrm{g}}=12 \mathrm{~Bq} \cdot \mathrm{m}^{-3}$ ); bassin parisien $\left(m_{g}=25\right.$ Bq. $\left.m^{-3}\right)$ par exemple. 


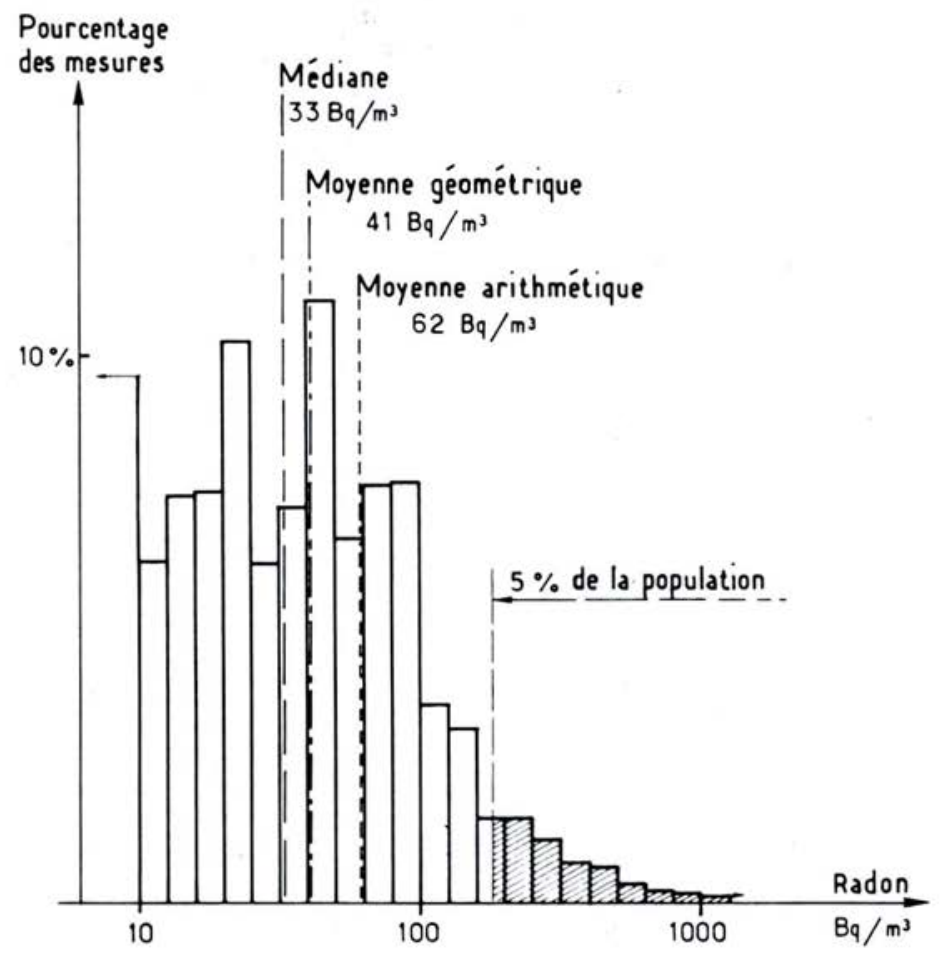

Fig. 2. - Histogramme de répartition des mesures de concentration en radon après pondération par la population.

A l'opposé, les départements à sols principalement granitiques connaissent les niveaux moyens les plus élevés $\left(>90\right.$ Bq. $\left.\mathrm{m}^{-3}\right)$ : Creuse $\left(m_{g}=147\right.$ Bq. $\left.m^{-3}\right)$, Corrèze $\left(m_{g}=107\right.$ Bq. $\left.m^{-3}\right)$, Loire $\left(m_{g}=105\right.$ Bq. $\left.m^{-3}\right)$ et Haute-Vienne $\left(m_{\mathrm{g}}=125\right.$ Bq. $\left.\mathrm{m}^{-3}\right)$ dans le Massif central, ou encore le Finistère $\left(m_{g}=99\right.$ Bq. $\left.m^{-3}\right)$ dans le Massif armoricain (fig. 3).

Ces variations géographiques reflètent, bien entendu, la teneur en uranium dans le sous-sol. L'uranium se rencontre en France de façon significative soit au sein des granitoïdes (cf. les gisements exploités en Vendée, Limousin, Forez par exemple), soit au sein des métamorphites de contact (les Bondons en Lozère), soit enfin en terrains sédimentaires détritiques (Lodève dans l'Hérault). Les teneurs moyennes des corps minéralisés varient de $0,1-0,2$ pour cent d'uranium à quelques 1 - 10 pour cent dans certaines formations particulièrement riches. 


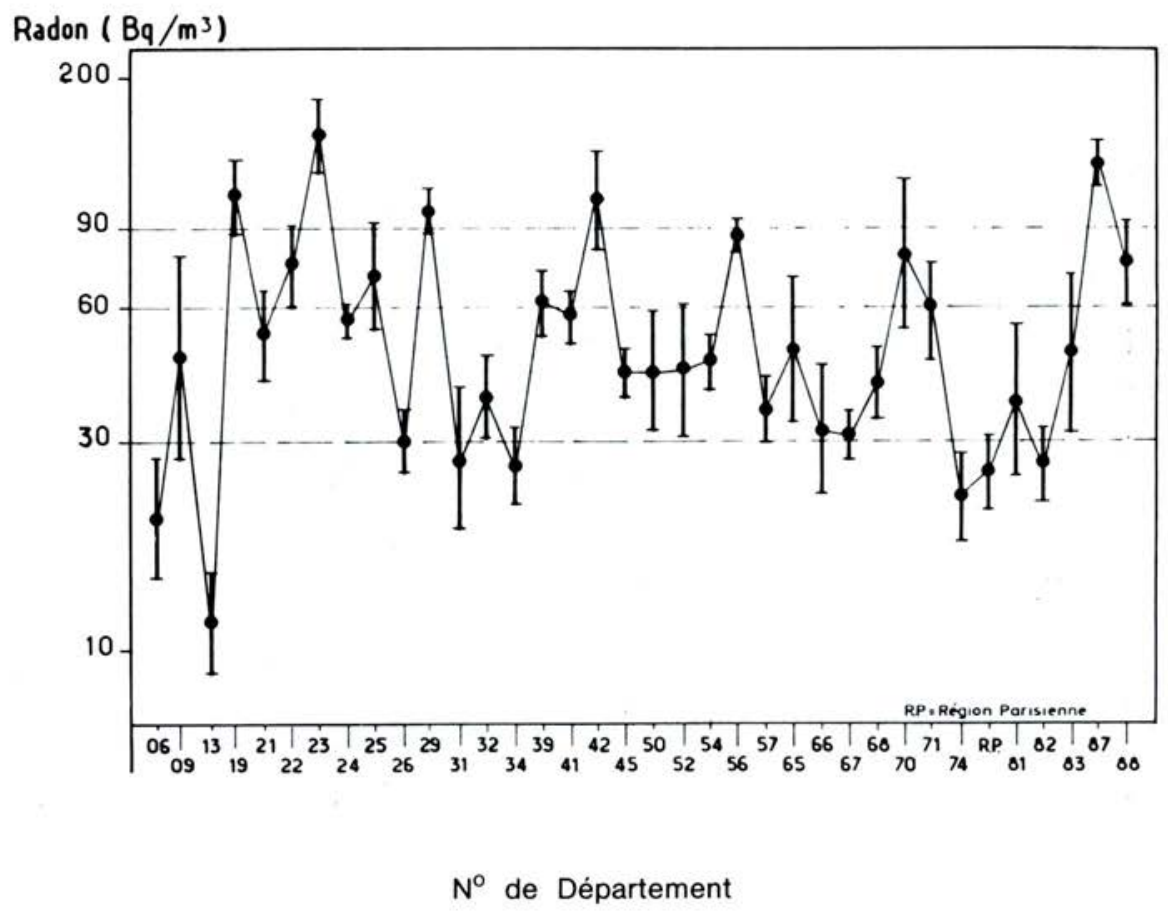

Fig. 3. - Répartition des moyennes géométriques avec leur intervalle de confiance calculées dans les différents départements.

Evidemment, les limites administratives de département ne constituent que très rarement celles d'unité géologique, et des variations géographiques sensibles peuvent être remarquées à l'intérieur de certains départements. C'est, par exemple, le cas très typique du département de la Haute-Vienne où la partie située au nord de la Vienne est largement dominée par une masse granitique riche en uranium comparée à la partie sud. Ainsi, la concentration moyenne en radon dans le nord est de l'ordre de 190 Bq. $\mathrm{m}^{-3}$ environ, tandis qu'elle est égale à $115 \mathrm{~Bq} \cdot \mathrm{m}^{-3}$ dans les maisons du sud du département. Les maisons construites quant à elles dans les communes se trouvant le long de la Vienne n'indiquent qu'une moyenne égale à $90 \mathrm{~Bq} \cdot \mathrm{m}^{-3}$. Cette plus faible valeur résulte probablement d'un apport local de sédiments exogènes moins riches en uranium, draînés par le fleuve. 


\section{A. RANNOU, G. TYMEN}

Cette discontinuité s'observe également dans le département de la Dordogne (fig. 4) ou encore dans les départements bretons [12].

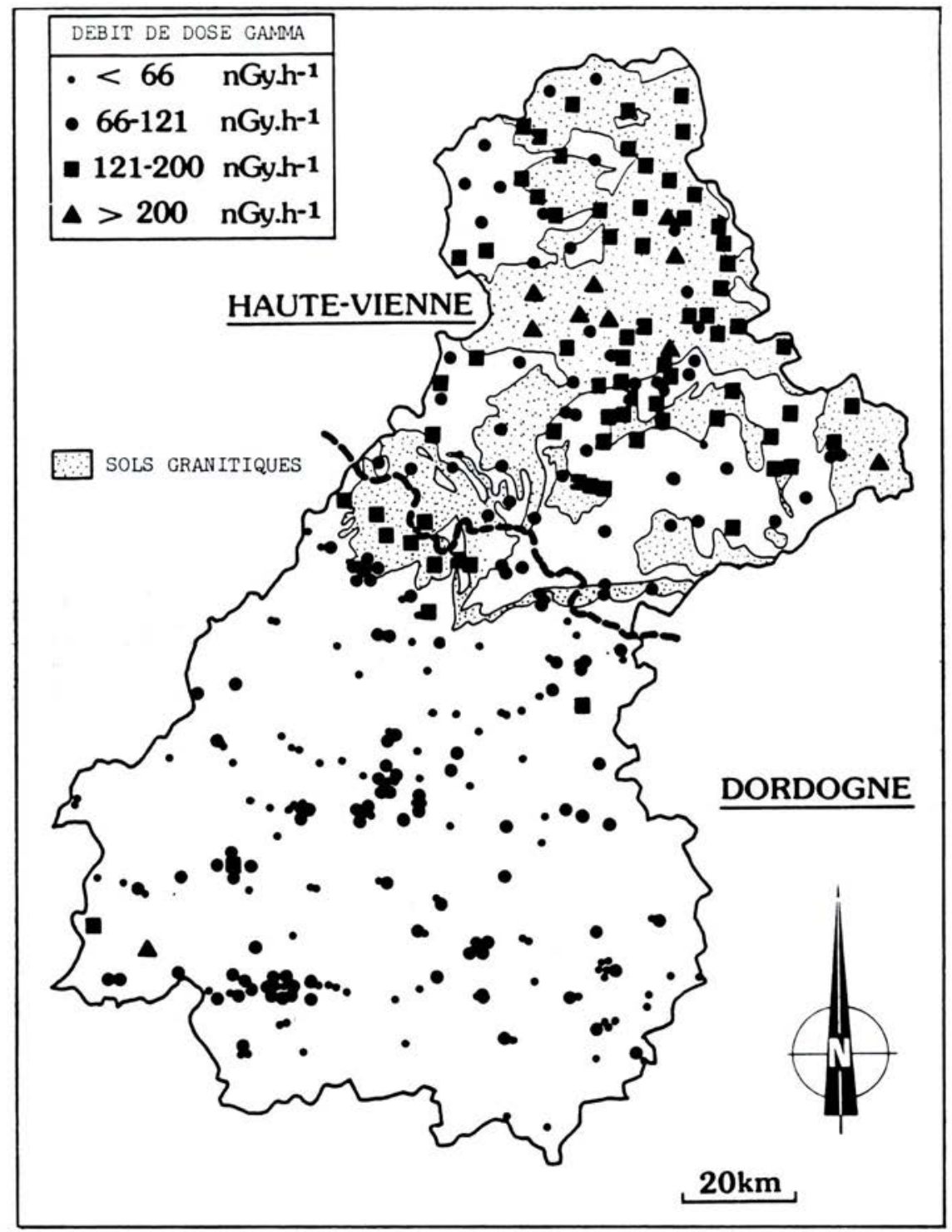

Fig. 4. - Mesures du débit de dose dû aux rayonnements gamma dans les maisons de Haute-Vienne et de Dordogne. 


\subsection{Rayonnements gamma terrestres}

La nature géologique des sols sous-jacents, et en particulier la teneur en uranium et thorium, est donc un facteur explicatif déterminant dans la problématique du radon. Une bonne corrélation entre la concentration en radon dans une maison donnée et le débit de dose dû aux rayonnements gamma terrestres pourrait intuitivement être espérée. En fait, l'analyse des deux paramètres mesurés dans une même maison montre un lien faible entre radon et rayonnements gamma. Pourtant, si l'on examine ces données plus en détail, par exemple pour le Morbihan (fig. 5), les observations suivantes peuvent être faites: aux niveaux de concentration en radon élevés, correspond un débit de dose annuel dû aux rayonnements gamma élevé. Inversement, à un faible débit de dose annuel dû aux rayonnements gamma correspondent des concentrations en radon nécessairement faibles.

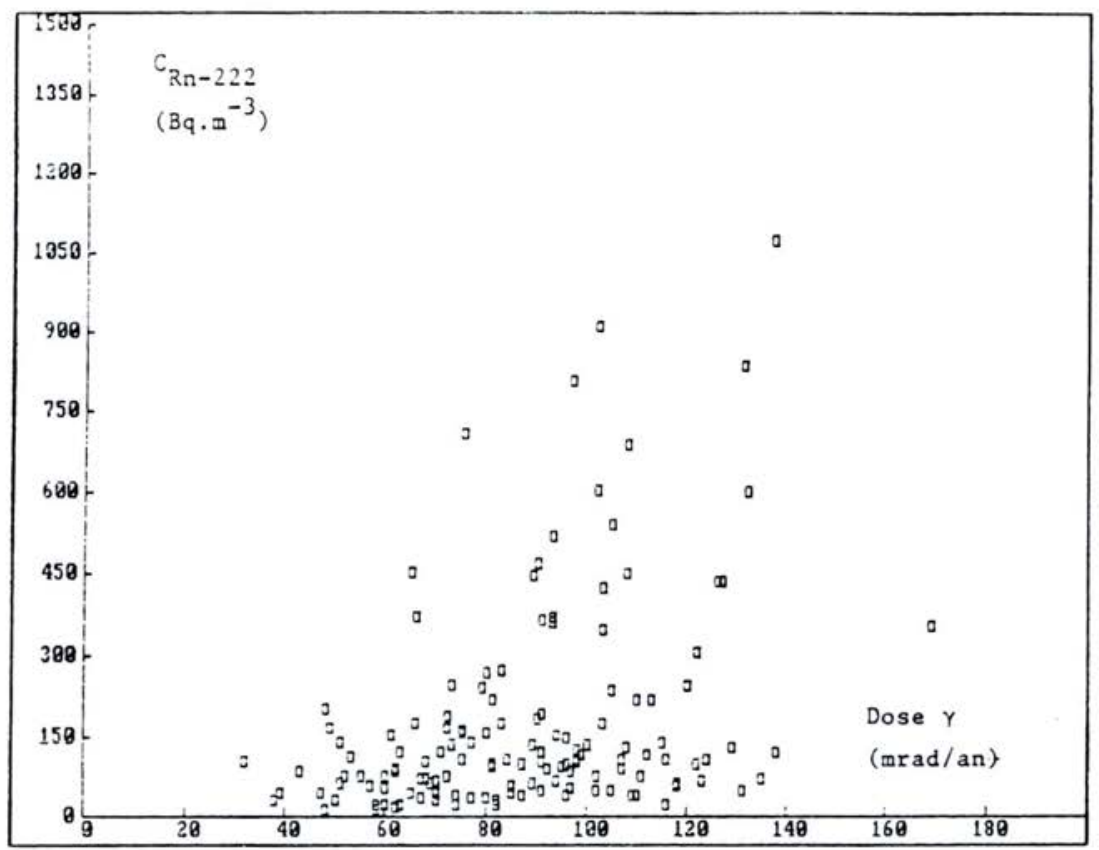

Fig. 5. - Mesure de la concentration en radon en fonction du débit de dose annuel dû aux rayonnements gamma mesuré dans les habitations du Morbihan.

Autrement dit, il apparaît que la présence d'un terrain riche en radioéléments naturels soit une condition nécessaire mais non suffisante pour que les maisons qui y sont construites présentent des teneurs importantes en radon. C'est le même constat qui ressort si l'on compare les deux paramètres à l'échelle de moyennes de départements (fig. 6). Ceci s'explique en partie par le fait que le radium qui constitue la source de radon dans 
les habitations n'est pas le seul radioélément naturel à l'origine du rayonnement gamma terrestre. L'existence des autres facteurs d'influence de la concentration en radon explique, pour le reste, cette assez faible corrélation.

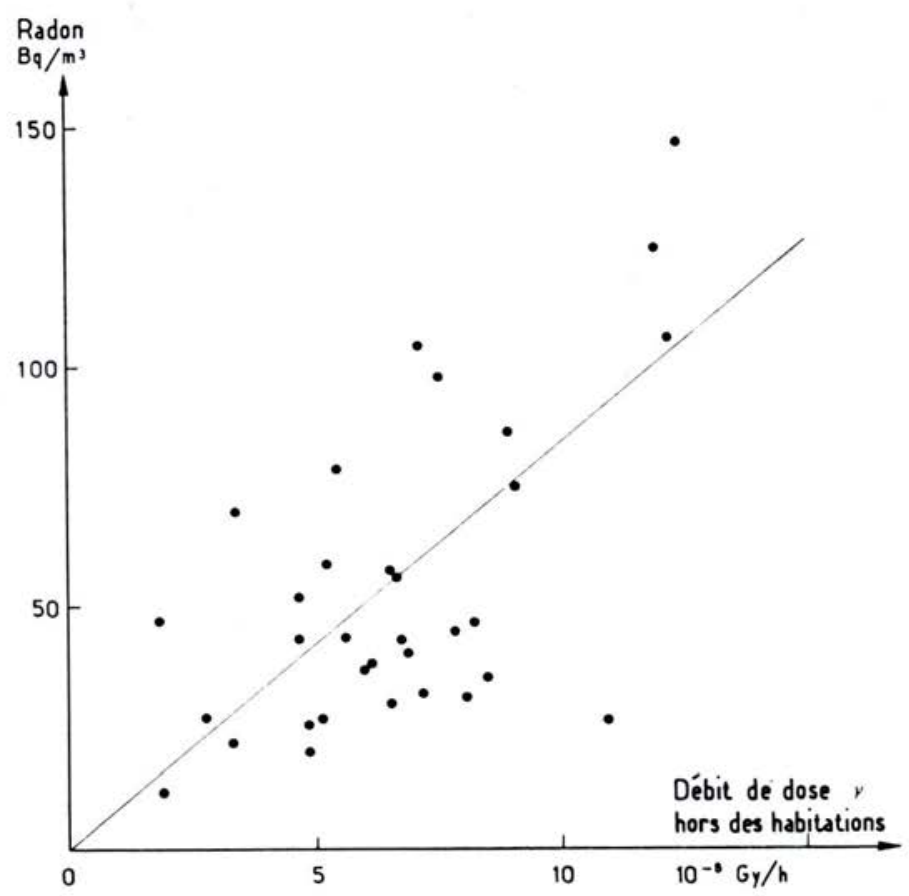

Fig. 6. - Comparaison des niveaux moyens de radon et de rayonnements gamma dans les différents départements.

Avant de présenter ici quelques résultats marquants qui mettent en évidence certains de ces facteurs explicatifs, nous allons tout d'abord rappeler les principales observations concernant l'étude régionale en Bretagne.

\subsection{Résultats de l'étude régionale en Bretagne}

\subsubsection{Protocole}

Seuls, les logements individuels de type moellon ou parpaing - quelques uns en béton - largement répandus dans les départements de l'Ouest, ont été pris en considération.

Les moyens déployés lors de la campagne du département du Morbihan, ont été plus importants que pour le Finistère (22 dosimètres actifs intégrés au lieu de 8 ), chaque participant recevant, à placer dans une pièce choisie par lui:

- un dosimètre actif intégré, pendant deux semaines d'exposition;

- un film passif pour la mesure du ${ }^{222} \mathrm{Rn}$, pendant deux semaines; 
- un film identique au précédent, exposé pendant une durée de trois mois;

- deux TLD pour la mesure de l'exposition gamma: l'un à l'intérieur, l'autre à l'extérieur, pendant trois mois également.

Par ailleurs, un questionnaire, susceptible de décrire les conditions expérimentales, était remis à chaque participant.

Au total, plus de 500 expériences significatives ont été réalisées pendant la période d'étude 1983-1987, constituant ainsi une base de données sans précédent à un tel échelon, et original de surcroît puisque associant en parallèle les techniques de dosimétrie active et passive.

\subsection{Résultats relatifs aux concentrations en énergie potentielle alpha}

Comme il a été dit au début de cet article, ce paramètre sert à caractériser l'exposition due à l'inhalation des descendants du ${ }^{222} \mathrm{Rn}$ et aussi du ${ }^{220} \mathrm{Rn}$, grâce à la possibilité qu'offre le dosimètre actif intégré CEA de discriminer en énergie les émetteurs alpha du radon et du thoron.

La représentation en histogramme des concentrations en EPA (fig. 7) pour les deux départements concernés présente des allures sensiblement analogues et confirme la dispersion déjà constatée à propos des variations des concentrations en radon. Afin d'examiner la répartition géographique des niveaux d'EPA, nous avons simplifié la représentation géologique d'une région extrêmement complexe, comme peut l'être le Massif armoricain, en ne considérant que trois types de sous-sol:

- les terrains granitiques, présents au nord et au sud de la Bretagne;

- les roches métamorphiques, présentes également au nord et au sud;

- et les terrains sédimentaires, plutôt situés au centre.
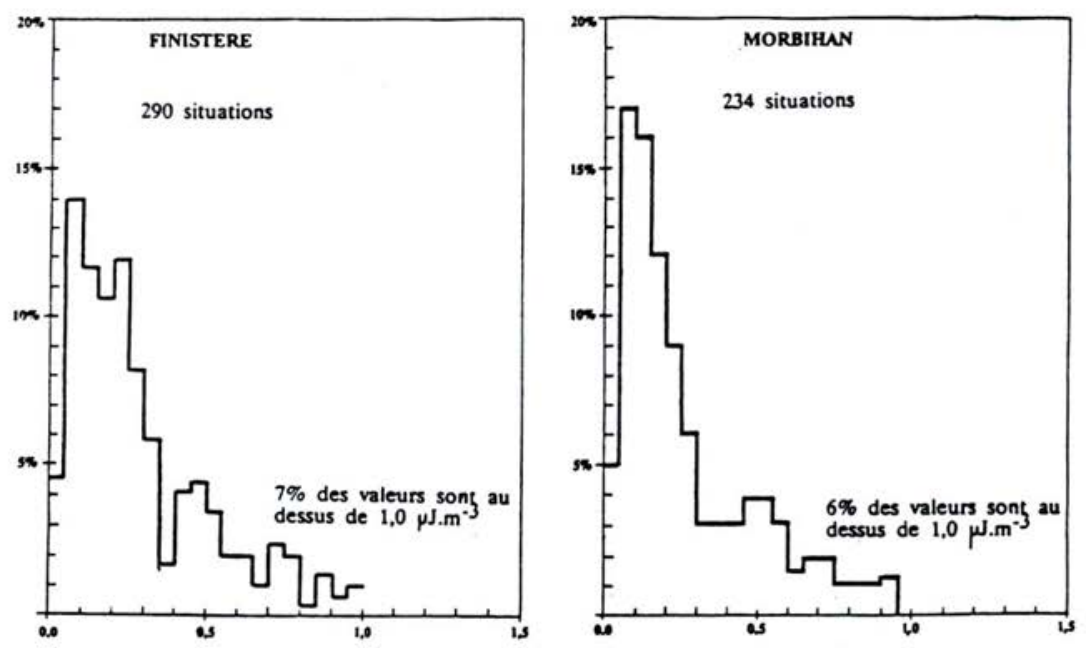

Fig. 7. - Histogrammes des variations des concentrations en énergie potentielle alpha totale en Bretagne. 
Les valeurs caractéristiques des distributions correspondant aux zones géologiques précédemment évoquées ont été portées dans le tableau I.

TABLEAU I Concentrations en énergie potentielle alpha totale $\left(\mu \mathrm{J} \cdot \mathrm{m}^{-3}\right)$ en Bretagne
en fonction de la nature du sol

\begin{tabular}{|c|c|c|c|c|c|}
\hline & $\begin{array}{l}\text { Nature } \\
\text { du sol }\end{array}$ & $\underset{\mathrm{me}}{\text { Nom }}$ & & Médiane & Moyenne \\
\hline \multirow{4}{*}{ 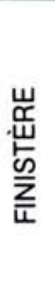 } & Granitique & $\begin{array}{l}\text { Nord } \\
\text { Sud }\end{array}$ & $\begin{array}{l}68 \\
77\end{array}$ & $\begin{array}{l}0,26 \\
0,27\end{array}$ & $\begin{array}{l}0,37 \\
0,39\end{array}$ \\
\hline & Métamorphique & $\begin{array}{l}\text { Nord } \\
\text { Sud }\end{array}$ & $\begin{array}{l}19 \\
22\end{array}$ & $\begin{array}{l}0,15 \\
0,48\end{array}$ & $\begin{array}{l}0,19 \\
0,60\end{array}$ \\
\hline & Sédimentaire & Centre & 99 & 0,19 & 0,33 \\
\hline & Total & & 285 & 0,25 & 0,35 \\
\hline \multirow{4}{*}{ 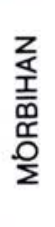 } & Granitique & & 98 & 0,22 & 0,32 \\
\hline & Métamorphique & & 43 & 0,25 & 0,35 \\
\hline & Sédimentaire & & 93 & 0,17 & 0,27 \\
\hline & Total & & 234 & 0,22 & 0,31 \\
\hline
\end{tabular}

On remarque que, dans l'ensemble, les secteurs à prédominance granitique ou métamorphique fournissent des CEPA plus élevées que les zones à dominante sédimentaire, quoique des valeurs assez élevées aient pu être relevées dans ces dernières. Par ailleurs, la contribution des descendants du thoron à l'énergie potentielle alpha totale varie dans des proportions plus ou moins importantes selon les situations, puisque dans cette étude, le rapport CEPA (Thoron) / CEPA totale peut prendre des valeurs comprises entre $5 \%$ et $90 \%$ ), la moyenne se situant vers $28 \%$. En général, lorsque l'énergie potentielle alpha est élevée, la contribution des descendants de ${ }^{222} \mathrm{Rn}$ l'emporte largement sur celle des descendants du ${ }^{220} \mathrm{Rn}$. Par contre, aux concentrations basses d'EPA, on trouve davantage de cas où la part due aux produits du ${ }^{220} \mathrm{Rn}$ équivaut ou l'emporte sur celle due aux descendants du ${ }^{222} \mathrm{Rn}$.

\subsubsection{Importance du facteur d'équilibre}

C'est un paramètre d'un intérêt majeur pour estimer les doses reçues par l'inhalation des descendants du radon. II est défini comme le rapport entre l'énergie potentielle alpha d'un mélange de produits de filiation du radon, et l'énergie potentielle alpha relative au mélange, s'il était en équilibre avec le parent, soit :

$$
F=\operatorname{CEPA}\left({ }^{222} \mathrm{Rn}\right)\left(\mathrm{J} . \mathrm{m}^{-3}\right) / 5,54.10^{-9} \mathrm{C}_{{ }^{222} \mathrm{Rn}}\left(\mathrm{Bq} \cdot \mathrm{m}^{-3}\right)
$$


Selon les auteurs, $F$ varie en général, en moyenne, entre 0,3 et 0,5. Expérimentalement, nous observons, dans cette étude régionale, des variations de $F$ entre 0 et 1 , la valeur moyenne se situant vers 0,34 pour le département du Morbihan et 0,39 pour le Finistère. Cette importante variabilité de $F$ est bien illustrée par la figure 8 qui représente les couples de points $F$ concentration en ${ }^{222} \mathrm{Rn}$, pour une même période de $15 \mathrm{j}$ d'exposition. On remarque, en particulier, que les fortes valeurs de $\mathrm{F}$ sont fréquemment associées à des niveaux de radon faibles ou moyennement faibles, mais jamais à des niveaux élevés. A l'opposé, à de fortes concentrations en radon correspondent des fractions d'équilibre généralement faibles. Cette importante dispersion des valeurs expérimentales de $\mathrm{F}$ pose le problème des relations de conversion exposition-doses quand, seules, les concentrations en radon sont disponibles.

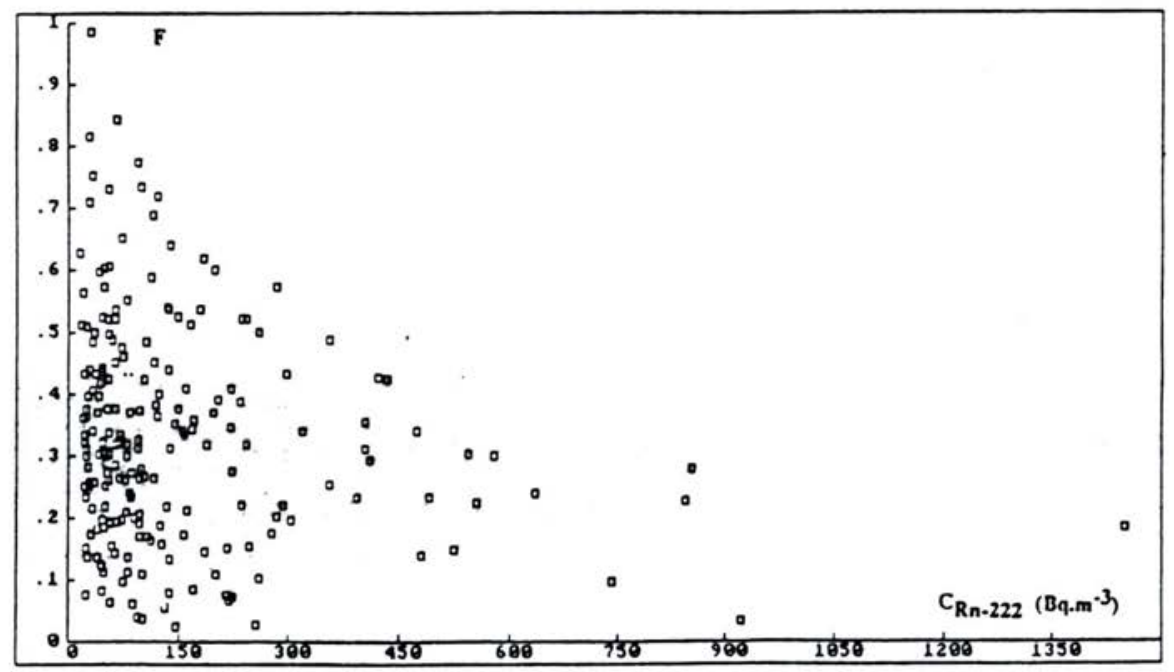

Fig. 8. - Données représentant les facteurs d'équilibre $F$ en fonction de la concentration en radon (Morbihan).

\subsection{Autres facteurs explicatifs}

\subsubsection{Variations saisonnières}

Nous n'avons pas traité directement le problème des variations saisonnières dans la campagne de mesure.

Les mesures de $15 \mathrm{j}$ effectuées dans le département du Morbihan semblent, toutefois, marquer une différence significative de niveaux en fonction du mois de l'année. Une corrélation ne doit pas pour autant être conclue dans la mesure où les résultats obtenus à un moment donné correspondent aussi à une série de communes d'un secteur donné du département. La différence peut donc simplement refléter des variations géographiques liées à la nature du sol. 
La figure 9 présente la comparaison des résultats obtenus dans les maisons du Morbihan, pour deux périodes d'exposition distinctes: la première de $15 \mathrm{j}$ et la seconde de 3 mois englobant la première période. Les résultats n'indiquent pas de différence significative au niveau des valeurs moyennes, que ce soit dans les maisons à concentration plutôt faible ou dans celles à concentration plutôt élevée; là encore, il ne s'agit pas de conclure qu'il n'existe pas de variations saisonnières mais plutôt que, en moyenne, la mesure de $15 \mathrm{j}$ s'avère suffisante même si moins représentative que celle de 3 mois. C'est aussi la conclusion faite par HERTZMAN et al. [3] pour qui des mesures de $30 \mathrm{j}$ sont apparues sans différence significative avec celles de $90 \mathrm{j}$. Les variations saisonnières ont toutefois été démontrées dans la plupart des pays et des rapports moyens entre mesure d'hiver et mesure d'été allant jusqu'à 2 ont été rapportés $[4,13]$.

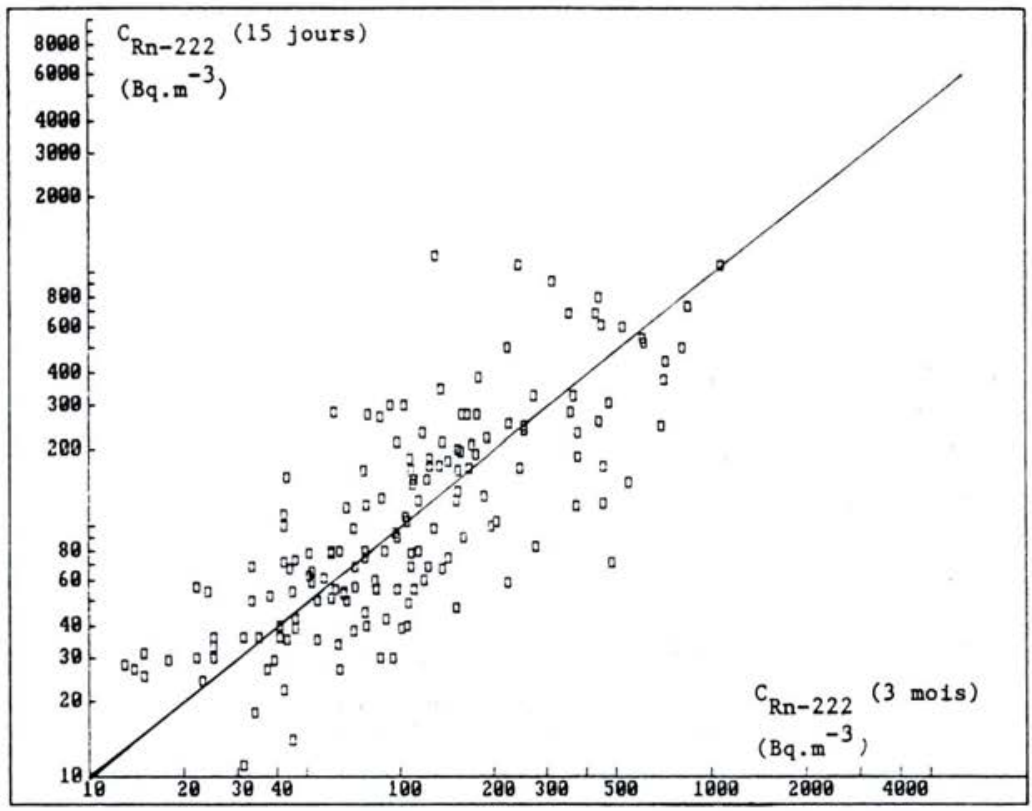

Fig. 9. - Comparaison des résultats de concentration en radon pour deux périodes différentes d'exposition.

\subsubsection{Localisation du détecteur dans la maison}

\subsubsection{L'étage}

Les résultats, classés en fonction de la pièce étudiée dans la maison, sont présentés dans le tableau II, pour la Creuse, la Corrèze, la Dordogne et la Haute-Vienne respectivement. Le classement, en fonction de l'étage, montre nettement des concentrations plus élevées au rez-de-chaussée qu'à l'étage. Le rapport moyen pour ces quatre départements est égal à $1,24 \pm 0,11$. 
RÉSULTATS DES CAMPAGNES DE MESURES DE RADON ET FACTEURS EXPLICATIFS

TABLEAU II

Moyennes géométriques des mesures de concentration en radon $\left(\mathrm{Bq} \cdot \mathrm{m}^{-3}\right)$ en fonction de la localisation de la pièce.

Le nombre de mesures est donné entre parenthèses.

A - CREUSE

\begin{tabular}{|l|c|c|c|}
\cline { 2 - 4 } \multicolumn{1}{c|}{} & Rez-de-chaussée & Etage & Total \\
\hline Séjour & $205(18)$ & $152(7)$ & $189(25)$ \\
\hline Chambre & $144(8)$ & $158(15)$ & $153(23)$ \\
\hline Total & $184(26)$ & $156(22)$ & $171(48)$ \\
\hline
\end{tabular}

B - CORRĖZE

\begin{tabular}{|l|c|c|c|}
\cline { 2 - 4 } \multicolumn{1}{c|}{} & Rez-de-chaussée & Etage & \multicolumn{1}{c|}{ Total } \\
\hline Séjour & $151(17)$ & $95(18)$ & $119(35)$ \\
\hline Chambre & $106(5)$ & $103(28)$ & $104(33)$ \\
\hline Total & $140(22)$ & $100(46)$ & $112(68)$ \\
\hline
\end{tabular}

C - DORDOGNE

\begin{tabular}{|l|c|c|c|}
\cline { 2 - 4 } \multicolumn{1}{c|}{} & Rez-de-chaussée & Etage & Total \\
\hline Séjour & $76(47)$ & $58(21)$ & $70(68)$ \\
\hline Chambre & $52(25)$ & $57(65)$ & $56(90)$ \\
\hline Total & $66(72)$ & $57(86)$ & $61(158)$ \\
\hline
\end{tabular}

\begin{tabular}{|l|l|c|c|c|}
\cline { 2 - 5 } \multicolumn{1}{c|}{} & Rez-de-chaussée & Etage & Total \\
\hline Séjour & $157(68)$ & $110(34)$ & $139(102)$ \\
\hline C - HAUTE-VIENNE & Chambre & $118(27)$ & $122(74)$ & $121(101)$ \\
\hline Total & $145(95)$ & $118(108)$ & $130(203)$ \\
\hline
\end{tabular}

En Bretagne aussi, des différences apparaissent statistiquement, selon que les mesures ont été faites au rez-de-chaussée ou à l'étage. Ces différences sont plus ou moins prononcées et parfois même contradictoires selon la nature du sol et les matériaux de construction (tableaux III A et III B). Dans le Morbihan, aucune différence significative ne se dégage pour les maisons en terrains sédimentaires, qu'elles soient construites en moellon ou en parpaing. Sur les terrains granitiques, au contraire, 
le rapport rez-de-chaussée/ étage est très nettement supérieur à 1 et maximal pour les maisons en moellon. Dans le Finistère, quelle que soit la nature du terrain, le rapport des moyennes est inférieur à 1 dans les maisons en moellon et très supérieur à 1 dans celles en parpaing.

TABLEAU III

\begin{abstract}
Rapport des moyennes des mesures de la concentration en énergie potentielle alpha au rez-de-chaussée et à l'étage en fonction de la nature du sol et des matériaux de construction.
\end{abstract}

\title{
A - FINISTĖRE
}

\begin{tabular}{|l|c|c|}
\cline { 2 - 3 } \multicolumn{1}{c|}{} & Moellon & Parpaing \\
\hline Granitique & 0,91 & 1,77 \\
\hline Sédimentaire & 0,83 & 2,23 \\
\hline
\end{tabular}

B - MORBIHAN

\begin{tabular}{|l|c|c|}
\cline { 2 - 3 } \multicolumn{1}{c|}{} & Moellon & Parpaing \\
\hline Granitique & 1,67 & 1,35 \\
\hline Sédimentaire & 1,14 & 1,00 \\
\hline
\end{tabular}

\subsubsection{Le type de pièce}

Si nous revenons au tableau II, des différences apparaissent avec des concentrations en moyenne légèrement plus élevées dans les salles de séjour que dans les chambres. La même constatation a été faite en Angleterre [13], aux Etats-Unis [2] ou encore au Danemark [10]. Nous expliquons, pour notre part, les niveaux plus importants mesurés dans les salles de séjour par le fait que celles-ci soient situées plutôt au rez-de-chaussée des maisons, tandis que les chambres se trouvent le plus souvent à l'étage.

En subdivisant les données selon le niveau de la pièce, il ressort de l'étude de ces quatre départements que:

- si les deux pièces se trouvent au rez-de-chaussée, alors la concentration est supérieure, en moyenne, dans la salle de séjour comparée à celle dans la chambre (rapport moyen $=1,41 \pm 0,06)$;

- si les deux pièces se trouvent à l'étage, alors la concentration est inférieure ou égale dans la salle de séjour, comparée à celle dans la chambre (rapport moyen $=0,95 \pm 0,05$ ).

Si cette observation a un sens, elle ne peut être attribuée qu'à une différence de ventilation ou de conception de la maison entre les deux situations analysées. 


\subsubsection{Matériaux de construction}

Les maisons en moellon, étudiées en Bretagne, présentent globalement les niveaux de concentration les plus élevés, comparativement à celles construites en parpaing: facteur 1,5 dans le Finistère et 1,1 dans les Côtes-du-Nord. La subdivision des données en groupes prenant en compte la pièce étudiée montre, cependant, des résultats plus nuancés, parfois même inattendus. L'analyse statistique se heurte ici à l'interférence de multiples co-facteurs, créant des situations où le paramètre d'influence recherché ne peut être isolé des autres facteurs.

\subsubsection{L'âge de la maison}

Les données relatives aux quatre départements du Centre sont classées dans le tableau IV, en fonction de la date de construction des maisons. En prenant 1948 comme date de référence (correspondant au grand "boom" de l'après-guerre, en matière de construction), les maisons anciennes ont des concentrations en moyenne supérieures à celles des maisons récentes, sauf en Dordogne. A contrario, il n'existe pas de différence systématique entre les résultats d'avant 1974 et ceux d'après 1974 (date correspondant aux mesures prises en construction pour économiser l'énergie). II est vrai que les maisons les plus anciennes ont pu, elles-mêmes, subir des rénovations visant à faire des économies d'énergie.

TABLEAU IV

Moyennes géométriques des mesures de concentration en radon $\left(\mathrm{Bq} \cdot \mathrm{m}^{-3}\right)$ en fonction de la date de construction.

Le nombre de mesures est donné entre parenthèses.

\begin{tabular}{|l|c|c|c|}
\cline { 2 - 4 } & avant 1948 & 1948 à 1974 & après 1974 \\
\hline \multirow{2}{*}{ CREUSE } & \multirow{2}{*}{$185(35)$} & $160(10)$ & $105(13)$ \\
\cline { 2 - 4 } & \multirow{2}{*}{ CORREZE } & après 1948: $125(27)$ \\
\hline \multirow{2}{*}{ DORDOGNE } & $126(56)$ & $84(13)$ & $127(10)$ \\
\cline { 2 - 4 } & \multirow{2}{*}{$59(125)$} & après 1948: $89(27)$ \\
\cline { 2 - 4 } & \multirow{2}{*}{$150(94)$} & après $1948: 60(108)$ \\
\cline { 2 - 4 } & \multirow{2}{*}{ HAUTE-VIENNE } & \multicolumn{2}{|c|}{ après $1948: 119(150)$} \\
\hline
\end{tabular}




\section{DISCUSSION ET CONCLUSION}

Les campagnes de mesure du radon dans les habitations en France ont permis de montrer des concentrations variant sur quatre ordres de grandeur. La distribution en données corrigées tenant compte de la densité de population ou de logements suggère une valeur médiane égale à $33 \mathrm{~Bq} \cdot \mathrm{m}^{-3}$. Les moyennes géométriques et arithmétiques sont égales à $41 \mathrm{~Bq} \cdot \mathrm{m}^{-3}$ et 62 Bq. $\mathrm{m}^{-3}$ respectivement. Selon nos estimations, $1,5 \%$ environ des logements actuels pourraient dépasser une concentration en radon de $400 \mathrm{~Bq} \cdot \mathrm{m}^{-3}$. L'étude par département montre des variations géographiques significatives liées à la nature géologique des sols. La recherche des régions ou zones les plus sensibles semble pouvoir s'appuyer sur ces considérations géologiques et sur la cartographie du territoire en rayonnements gamma telluriques.

La base de données qui se constitue a déja permis de mettre en évidence l'influence de quelques paramètres liés à l'habitat. L'analyse statistique s'avère cependant difficile, ces différents paramètres étant le plus souvent liés entre eux. Des variations, parfois importantes, peuvent être relevées dans une même habitation, signe de la prudence qu'il faut donner à l'annonce d'un résultat unique.

Enfin, certains facteurs explicatifs devront encore être étudiés pour aider aussi à la recherche de méthodes préventives ou curatives: ventilation, revêtements sur les murs, type de fondation, etc...

\section{RÉFÉRENCES}

[1] CHAPUIS A.M., DAJLEVIC D., DUPORT P., SOUDAIN G. - Dosimétrie du radon, in: 8th Int. Conference on Nuclear photoghraphy and solid state nuclear track detectors, Bucarest, Jul. 10-15, 1972 (NICOLAE M., Ed.). Bucarest: Institute of atomic physics, 1972, vol. 2, 319-325.

[2] COHEN B.L. - A national survey of ${ }^{222} \mathrm{Rn}$ in US homes and correlating factors. Health Phys., 1986, 51 (2) 175-183.

[3] HERZMAN S., SAMUELSSON C. - The use of open track films for ${ }^{222} \mathrm{Rn}-$ measurements in dwellings. In: Solid state nuclear track detectors, 13. International conference, Rome, 23-27 Sept. 1985. Nucl. Tracks Radiat. Meas., 1986, 12 (1/6) 755-758:

[4] McGREGOR R.G., WALKER W.B., LETOURNEAU E.G. - Radon and radon daughters levels in energy efficient housing. Sci. Total Environ., 1985, 45, 271-278.

[5] MCLAUGHLIN J.P. - An assessment of indoor radon exposure in Ireland, in: Proceedings of the Second APCA international specialty conference on indoor radon, Cherry Hill, Philadelphia, April 6-10, 1987.

[6] MADELMONT C., JEANMAIRE L., RANNOU A., RENOUARD H., VERRY M. Programme de mesures de l'exposition naturelle aux rayonnements ionisants en France. 1. Description des méthodes et mise en œuvre. Bull. Acad. Vét. France, $1984,57,358-368$. 
[7] POSTENDORFER J. - Indoor radon exposure in the Federal Republic of Germany, in: Proceedings of the Second APCA international specialty conference on indoor radon, Cherry Hill, Philadelphia, April 6-10, 1987.

[8] PUT L.W., de MEIJER R.J., and BOSNJAKOVIC B.F.M. - Radon in the Netherlands, in: Proceedings of the Second APCA international specialty conference on indoor radon, Cherry Hill, Philadelphia, April 6-10, 1987.

[9] RANNOU A., JEANMAIRE L., TYMEN G., MOUDEN A., NAOUR E., PARMENTIER N., RENOUARD $\mathrm{H}$. - Use of cellulose nitrate as radon and radon daughters detectors for indoor measurements. Nuclear Tracks, 1986, 12 (1/6) 747-750.

[10] SORENSEN A., BOTTER-JENSEN L., MAJBORN B., NIELSEN S.P. - A pilot study of natural radiation in Danish homes. Sci. Total Environ., 1985, 45, 351-356.

[11] SWEDJEMARK G.A. - Radon and its decay products in housing, Doctoral dissertation, Stockholm, 1985.

[12] TYMEN G., MOUDEN A., RANNOU A., MADELMONT C., PARMENTIER N. Results of natural radon daughter exposure measurements by using alpha dosemeters in western individual houses of France. In: Solid state nuclear track detectors, 13. International conference, Rome, 23-27 Sept. 1985. Nucl. Tracks Radiat. Meas., 1986, 12 (1/6) 751-754.

[13] WRIXON A.D., GREEN B.M.R., LOMAS P.R., MILES J.C.H., CLIFF K.D., FRANCIS E.A., DRISCOLL C.M.H., JAMES A.C., O'RIORDAN M.C. - Natural radiation exposure in U.K. dwellings. NRPB-R-190, 1988. 